\title{
Ärztliche Aufklärungspflichten bei diagnostischen Röntgenuntersuchungen,
} 2. Teil

\section{Der 1. Teil dieses Beitrages beschäftigte sich mit den Pflichten des Arztes aus dem Behandlungsvertrag im Bereich der Aufklä- rung und der Eingriffsqualität ionisierender Strahlen (vgl. Fort- schr Röntgenstr 2016, DRG-Mitteilungen S. 218 ff.). Der hier folgende 2. Teil setzt sich mit den Fragen der Delegationsfähig- keit der Aufklärung in der Radiologie auseinander und benennt zusammenfassend die Vorgaben, die vom Radiologen im Zu- sammenhang mit der Aufklärung des Patienten, der sich einer Röntgenuntersuchung unterzieht, zu beachten sind.}

\section{Delegationsfähigkeit der Aufklärung}

In den Bereichen interventioneller und minimalinvasiver radiologischer Verfahren oder bei CT- und MRT-Untersuchungen mit Kontrastmittelinjektion besteht regelmäßig eine Pflicht zur Risikoaufklärung durch den Arzt, da es sich um körperliche Eingriffe handelt, in die der Patient wirksam einwilligen muss. Demgegenüber besteht bei Röntgenuntersuchungen in der Regel keine Aufklärungspflicht gegenüber dem Patienten, mit Ausnahme der Vorgaben nach der RöV, insbesondere nach den $\S \S 23 \mathrm{ff}$.

In der Praxis stellt sich jedoch zunehmend die Frage, ob und ggfls. in welchem Umfang die Aufklärung von einer MTRA durchgeführt werden darf.

\section{Geltung des Arztvorbehalts}

Die bisherige Rechtsprechung hat sich zu der Delegationsfähigkeit der Risikoaufklärung auf nicht ärztliche Fachkräfte eindeutig geäußert. Der Aufklärung des Patienten kommt eine elementare Aufgabe der ärztlichen Tätigkeit zu, wie die Rechtsprechung durch eine Vielzahl von Entscheidungen dokumentiert hat. Die Aufklärung gehört danach zum Kernbereich der ärztlichen Tätigkeit (BGH NJW 1974, 604; vgl. daneben OLG Brandenburg, Urt. v. 27.03.2008, Az.: 12 U 239/06; OLG Jena NJW-RR 2006, 135, OLG Celle VersR 1981, 1184). Im juristischen Sinne kann die Aufklärung daher nicht an nicht ärztliche
Fachkräfte delegiert werden und muss immer von einem Arzt durchgeführt werden. Dies beruht auch auf der Notwendigkeit, dass der Patienten nicht nur über die mit der Therapie oder Untersuchung selbst einhergehenden, sondern auch über mittelbare Umstände und Folgen aufzuklären ist. Im Rahmen der von dem Arzt vorzunehmenden Grundaufklärung über Art- und Schweregrad des Eingriffs ist es in aller Regel erforderlich, dass der Patient auch einen Hinweis auf das schwerste, möglicherweise in Betracht kommende Risiko erhält (BGH NJW 1991, 2346, 2347). Dem Arzt ist zwar grundsätzlich in den Diagnostik- und Therapiemethoden ein freies Ermessen eingeräumt (BGH NJW 1989, 1538), bei risikobehafteten diagnostischen Methoden hat der Arzt allerdings eine besonders sorgfältige Güterabwägung zwischen der erwarteten Aussagefähigkeit, den Klärungsbedürfnissen und den besonderen Risiken für den Patienten vorzunehmen. Umfang und $\mathrm{Ge}-$ nauigkeit der erforderlichen Aufklärung des Arzts sind umgekehrt proportional zur Dringlichkeit und dem Dringlichkeitsgrad des medizinischen Eingriffs und seiner Heilungsaussicht. Gleichzeitig ist eine Aufklärung über Abwarten, Nichtstun oder alternative Behandlungsmethoden geboten.

Der Gesetzgeber hat diese Vorgaben der Rechtsprechung in $\S 630$ e Abs. 1 BGB entsprechend umgesetzt. Danach ist „der Behandelnde" verpflichtet, den Patienten über sämtliche für die Einwilligung wesentlichen Umstände aufzuklären. Aus

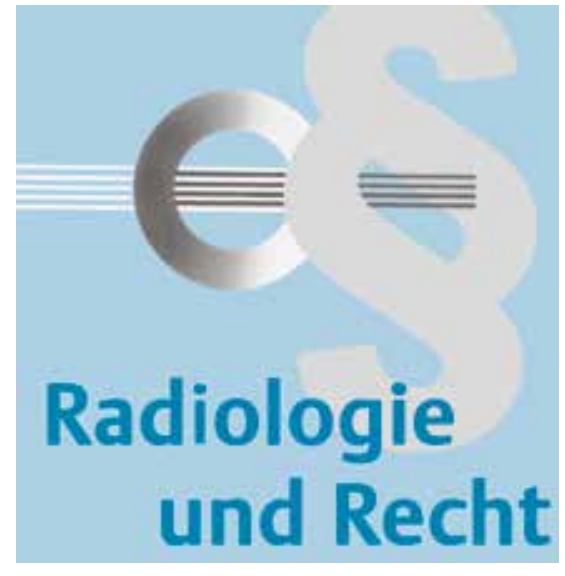

der Gesetzesbegründung zum Patientenrechtegesetz ist hierzu folgendes zu entnehmen:

„Wird der Eingriff durch einen Arzt vorgenommen, hat die Aufklärung durch einen Arzt zu erfolgen. Insbesondere bei „Individuellen Gesundheitsleistungen" (IGeL) ist in der Praxis festzustellen, dass viele dieser Angebote bereits z. B. bei der Anmeldung in der Arztpraxis von Arzthelferinnen und Arzthelfern den Patientinnen und Patienten unterbreitet werden. [...] Dies führt zunehmend zu Irritationen und Verunsicherungen von Patientinnen und Patienten, wie insbesondere ältere Verbraucherinnen und Verbraucher immer wieder auf Verbraucherkonferenzen in den letzten Jahren beklagten, da für diesen Personenkreis das Aufklärungsgespräch zwischen Arzt und Patient einen besonders hohen Stellenwert hat. Sollte der behandelnde Arzt die Aufklärung nicht selbst durchführen, so hat er die Information des Patienten durch eine Kollegin oder einen Kollegen so zu organisieren, dass sie voll gewährleistet ist. Eine Delegation der Aufklärung an weiteres ärztliches Personal (z. B. Arzthelferinnen oder Arzthelfer) wird durch die Erweiterung der Formulierung im Vorschlag durch die Voraussetzungskriterien der gleichen fachlichen Befähigung und Qualifikation in der Regel ausgeschlossen.“

(vgl. Entwurf eines Gesetzes zur Verbesserung der Rechte von Patientinnen und Patienten vom 15.08.2012, BT-Drucksache 17/10488, Stellungnahme des Bundesrates, Zu Artikel 1 Nummer 4 (§ 630e Absatz 2 Satz 1 Nummer 1 BGB), S. 42) 
Beteiligungsmöglichkeiten von nicht ärztlichem Fachpersonal

Die gesetzliche Verpflichtung die Behandlungs- und Risikoaufklärung durch einen Arzt vornehmen zu lassen, führt zu der Frage, in welchen Fällen eine Möglichkeit der Beteiligung von MTRA oder MFA an diesem Teil der ärztlichen Behandlung besteht.

In diesem Zusammenhang stellt sich zunächst die Frage, welche Anforderungen an das qualifizierte medizinisch-technische Fachpersonal zu stellen sind, das bei der Anwendung technisch mitwirken darf.

Nach $§ 24$ Abs. 2 Nr. 1 oder 2 RöV ist zur technischen Durchführung radiologischer Untersuchungen nur qualifiziertes medizinisch-technisches Fachpersonal berechtigt, namentlich entweder gemäß § 24 Abs. 2 Nr. 1 RöV eine Person „mit einer Erlaubnis nach $\S 1$ Nr. 2 des MTA-Gesetzes“ (Medizinisch-technische Radiologieassistenten; im Folgenden: MTRA) oder gemäß $\S 24$ Abs. 2 Nr. 2 RöV eine Person „mit einer staatlich geregelten, staatlich anerkannten oder staatlich überwachten abgeschlossenen Ausbildung, wenn die technische Durchführung Gegenstand ihrer Ausbildung und Prüfung war und sie die erforderliche Fachkunde im Strahlenschutz" besitzt (i.d.R. Medizinisch-technische Assistenten mit entsprechender Zusatzqualifikation und Fachkunde im Strahlenschutz gemäß § 18a Abs. 1 und 2 RöV; im Folgenden: MTA mit Zusatzqualifikation).

Personen, wie medizinische Fachangestellten (MFA), die über eine sonstige medizinische Ausbildung im Sinne des § 24 Abs. 2 Nr. 4 RöV verfügen, können die Fachkunde dagegen nicht erwerben. Die unter Nr. 4 genannten Personen verfügen lediglich über eine sonstige medizinische Ausbildung und die erforderlichen Kenntnisse im Strahlenschutz. Die erforderlichen Kenntnisse werden in der Regel durch eine für das jeweilige Anwendungsgebiet geeignete Einweisung und praktische Erfahrung erworben ( $\S 18 \mathrm{a}$ Abs. 3 Satz 1 RöV) und bleiben daher hinter der Fachkunde zurück. Die fehlende Fachkunde bei Personen nach § 24 Abs. 2 Nr. 4 RöV wird dadurch kompensiert, dass diese Personen nur „unter ständiger Aufsicht und Verantwortung" eines fachkundigen Arzts tätig werden dürfen. Den mit bestimmten Tätigkeiten verbundenen Risi- ken ist durch eine entsprechend intensive Aufsicht des fachkundigen Arzts zu begegnen.

Während einer durchgeführten Röntgenuntersuchung hat der anwesende, verantwortliche Radiologe daher die Wahl, die Untersuchung technisch entweder selbst durchzuführen (§ 24 Abs. 2 i.V.m. Abs. 1 RöV) oder einem qualifizierten medizinisch-technischen Fachpersonal (MTRA oder MTA mit Zusatzqualifikation) zur selbständigen technischen Durchführung zu übertragen (§ 24 Abs. $2 \mathrm{Nr}$. 1 oder 2 $\mathrm{RöV}$ ) oder aber unter seiner ständigen Aufsicht und Verantwortung entweder Ärzte ohne entsprechende Fachkunde, aber mit entsprechenden Kenntnissen im Strahlenschutz (§ 24 Abs. 2 i.V.m. Abs. 1 Nr. 3 RöV) oder Personen mit einer abgeschlossenen sonstigen medizinischen Ausbildung mit den erforderlichen Kenntnissen im Strahlenschutz ( $§ 24$ Abs. $2 \mathrm{Nr}$. 4 RöV) oder Auszubildende im fachlich einschlägigen Bereich (§ 24 Abs. 2 Nr. 3 RöV) unselbständig einzusetzen

Zwar dürfen Medizinische Fachangestellte daher ohne Einschränkungen hinsichtlich der technischen Durchführung wie Ärzte oder MTRA tätig werden. Allerdings dürfen sie nur unter ständiger Aufsicht und Verantwortung eines fachkundigen Arzts tätig werden. Bei dem nicht ärztlichen Personal nach § 24 Abs. 2 RöV kann davon ausgegangen werden, dass es den Arzt unter den dort genannten Voraussetzungen, im Zusammenhang mit der Stellung der rechtfertigenden Indikation und den hiermit zusammenhängenden Verpflichtungen nach den $\S \S 23 \mathrm{ff}$. RöV fachlich unterstützen kann.

Dies gilt einerseits für die Feststellung der patientenspezifischen Parameter, die der Arzt nach $\S 23$ Abs. 2 und 3 RöV bei dem Patienten vor der Durchführung der Untersuchung zu erfragen hat. Diese Informationen können von den Personen nach $\S 24$ Abs. 2 RöV im Rahmen der Vorbereitung der technischen Durchführung der Untersuchung abgefragt werden.

Auch ist es zulässig, dass dieses qualifizierte Personal dem Patienten Merkblätter übergibt und erläutert, die allgemeine Hinweise und Verhaltenspflichten im Zusammenhang mit der Durchführung der Röntgenuntersuchung sowie individuelle Fragen zum Gesundheitszustand des Patienten, einschließlich der röntgenspezifi- schen Risikofaktoren, enthält. Das Personal kann diese Patienteninformationen auch erfragen und für den Arzt dokumentieren.

Für den Fall des Bestehens von individuellen Risiken auf Seiten des Patienten ist der Arzt jedoch, nach den oben erfolgten Ausführungen verpflichtet, den Patienten persönlich mündlich aufzuklären. Ergänzend ist an dieser Stelle darauf hinzuweisen, dass Aufklärungsformulare keinen Ersatz für das persönliche Aufklärungsgespräch darstellen, sondern dieses nur ergänzen können (vgl. BGH NJW 1985, 1399).

\section{Aufklärung durch MTRA bei Kont- rastmittelapplikation?}

Die Rechtsprechung ist mittlerweile der Auffassung, dass Injektionen zur Kontrastmittelgabe durch eine MTRA generell zulässig sind (vgl. OLG Dresden, Urt. v. 24.07.2008, Az.: 4 U 1857/07; Amtsgericht Karlsruhe, Urt. vom 04.04.1997, Az.: 13 C 448/95). Zwar stellt eine derartige Injektion einen körperlichen Eingriff dar, dessen Vornahme prinzipiell in den Verantwortungsbereich des Arzts gehört. Es handelt sich hierbei aber nicht um eine Tätigkeit, die aufgrund der Schwierigkeit, Gefährlichkeit oder Unvorhersehbarkeit zwingend von einem Arzt erbracht werden muss.

Im Rahmen der Berufsausbildung zur MTRA, werden die für die Verabreichung einer Injektion erforderlichen medizinischen Grundlagenkenntnisse vermittelt. Nach $\S 3$ Nr. 2 des Gesetzes über die technische Assistenten in der Medizin (MTAG) soll die Ausbildung zum radiologischen MTA u.a. dazu befähigen, unter Anwendung geeigneter Verfahren in der radiologischen Diagnostik bei der Erkennung und Behandlung von Krankheiten in der Strahlentherapie und Nuklearmedizin mitzuwirken. Die Vermittlung dieser Kenntnisse erfolgt innerhalb einer 3-jährigen Ausbildung, die u.a. eine praktische Tätigkeit in einem Krankenhaus oder einer gleichgerichteten Einrichtung beinhaltet $(\S 4$ MTAG). Nach § 1 Abs. 2 der Ausbildungsund Prüfungsordnung für technische Assistenten in der Medizin (MTA-APrV) sind die Schüler während dieses 6-wöchigen Ausbildungsabschnitts in Verrichtungen und Fertigkeiten praktisch zu unterweisen, die für ihre Berufstätigkeit von Bedeutung sind. Nach Abschluss der Ausbil- 
dung ist es einem MTA gemäß $\S 9$ Abs. 3 MTAG einem MTA gestattet, auf ärztliche Anordnung Tätigkeiten auszuüben, deren Ergebnisse der Erkennung einer Erkrankung und der Beurteilung ihres Verlaufs dienen. Zudem besagt § 9 Abs. 1 Nr. 2 a), dass nur eine MTRA zur „Durchführung der technischen Arbeiten und Beurteilung ihrer Qualität in der Radiologischen Diagnostik und anderen bildgebenden Verfahren einschließlich Qualitätssicherung“ berechtigt ist. Es entspricht daher dem gesetzgeberischen Leitbild, dass einfache und mit nur geringen Risiken verbundene Injektionen einer MTRA übertragen werden können.

Diese Einschätzung entspricht auch den Vorgaben der Vereinbarung über die Delegation ärztlicher Leistungen an nichtärztliches Personal in der ambulanten vertragsärztlichen Versorgung gemäß $\S$ 28 Abs. 1 S. 3 SGB V vom 1. Oktober 2013 (vgl. Anhang zur Anlage 24 des BMV-Ä).

Demgegenüber dürfte es, hinsichtlich der Notwendigkeit der Aufklärung der Risiken über die Kontrastmittelgabe selbst, dabei bleiben, dass hierüber der Arzt den Patienten persönlich aufzuklären hat. Hintergrund ist, dass eine nicht ärztliche Fachkraft, wie eine MTRA, nur punktuell aufklären kann und nicht umfassend. Umfassend kann jedoch nur derjenige aufklären, dessen Kenntnisse und Erfahrungen über den Bereich hinausgehen, die für die anstehende Untersuchung relevant sind. Dies gilt auch deshalb, weil sich während des Aufklärungsgesprächs herausstellen kann, dass medizinische Gründe der anstehenden Untersuchung entgegenstehen, die von einer nicht ärztlichen Fachkraft u.U. nicht erkannt werden können.

Horizontale und vertikale Arbeitsteilung im Bereich der Aufklärung Daneben ist es aber denkbar, dass der Arzt die Aufklärung auf einen anderen Arzt überträgt. § 630e Abs. 2 Nr. 1 BGB sieht insoweit vor, dass die Aufklärung durch den Behandelnden oder durch eine Person erfolgt, „die über die zur Durchführung der Maßnahme notwendige Ausbildung verfügt." Nach der Gesetzesbegründung zu § 630e BGB wird dabei auch die Möglichkeit einer vertikalen arbeitsteiligen Aufklärung zwischen unterschiedlichen Fachgebiete im Sinne einer Stufenaufklärung durch das Gesetz ermöglicht (vgl.
Entwurf eines Gesetzes zur Verbesserung der Rechte von Patientinnen und Patienten v. 15.08. 2012, BT-Drucksache 17/10488, Zu § 630e (neu), S. 24):

„Primär hat derjenige, der die Maßnahme durchführt, selbst den Patienten aufzuklären. Daneben soll es aber auch möglich sein, die Aufklärung durch eine andere Person vornehmen zu lassen, allerdings vorausgesetzt, sie verfügt über die zur sachgerechten Aufklärung notwendige Befähigung und damit über die für die Durchführung der Maßnahme adäquate fachliche Qualifikation. Folglich muss beispielsweise der Arzt, der einen operativen Eingriff durchführt, nicht mit der Person des Aufklärenden identisch sein. Die aufklärende Person muss allerdings die notwendige Befähigung und Qualifikation zur Durchführung der Operation besitzen. Dies hat zur Folge, dass die Aufklärung für gesonderte Maßnahmen unter Umständen jeweils gesondert erfolgen muss. So hat etwa der Operateur über die Risiken der Operation einschließlich des mit der Operation verbundenen Risikos und ein Anästhesist über die Risiken der Narkose aufzuklären.“

Stufenaufklärung kann auch bedeuten, dass Teile der Aufklärung die allgemeine medizinische Risiken beinhalten und daher von jedem Arzt beantwortet werden können, an andere Ärzte delegiert werden. Dies kann z.B. Fragen nach Allergien, Nierenfunktionen oder Schilddrüsenfunktionen beinhalten. Insoweit kann auch bei radiologischen Untersuchungen arbeitsteilig über die allgemeinen Risiken einer Therapie durch den behandelnden oder überweisenden Facharzt und über die eingriffsbedingten Risiken in der Radiologie durch den Radiologen aufgeklärt werden.

Die Aufklärungspflicht kann ein Arzt danach zwar einem anderen Arzt übertragen. $\mathrm{Zu}$ beachten ist jedoch, dass in diesen Fällen eine Haftung für Aufklärungsversäumnisse weiterhin den behandelnden Arzt treffen kann. Die Übertragung der Aufklärung auf einen anderen Arzt entlastet diesen nicht von der vertraglichen Haftung für den ihm nachgeordneten Arzt, seinen sog. Erfüllungsgehilfen und nicht ohne weiteres von der deliktischen Haftung. Wenn der behandelnde Arzt entschuldbar eine wirksame Einwilligung des Patienten angenommen hat, kann sei- ne Haftung für nachteilige Folgen der Behandlung wegen fehlender Rechtswidrigkeit seines Verhaltens entfallen (BGH, Urteil vom 23.09.1975 - VI ZR 232/73). Voraussetzung hierfür ist, dass der Irrtum des Behandlers nicht auf Fahrlässigkeit beruht. Fahrlässigkeit ist im Sinne des $§ 276$ Abs. 1 S. 2 BGB die Außerachtlassung der im Verkehr erforderlichen Sorgfalt. Diese wird bei einer Übertragung der Aufklärung auf einen anderen Arzt nur dann zu verneinen sein, wenn der nicht selbst aufklärende Arzt durch geeignete organisatorische Maßnahmen und Kontrollen sichergestellt hat, dass eine ordnungsgemäße Aufklärung durch den damit betrauten Arzt gewährleistet ist. Der Arzt, der nicht selbst aufklärt, muss daher eine die Aufklärung sicherstellende Struktur schaffen, erhalten und stichprobenartig überwachen (OLG Karlsruhe, Urt. v. 19.03.1997 13 U 42/96).

\section{Ergebnis und Schlussfolgerungen $\nabla$}

Ausgehend von den bisherigen Ausführungen ergeben sich aus dem Zusammenspiel zwischen den ärztlichen Pflichten aus dem Behandlungsvertrag nach $\S \S$ 630a BGB und den Anwendungsgrundsätzen nach $\S \S 23$ ff. RöV für den Radiologen folgende Vorgaben im Zusammenhang mit der Aufklärung des Patienten, der sich einer Röntgenuntersuchung unterzieht:

1. Die zivilrechtliche Haftung eines Arztes aufgrund einer fehlenden Aufklärung und dem Auftreten einer Schädigung setzt einen nachweisbaren Kausalzusammenhang voraus.

2. Der Nachweis einer pathologischen Gesundheitsbeeinträchtigung, insbesondere einer Krebserkrankung, kann angesichts der geringen Strahlendosen, die bei ordnungsgemäß durchgeführten röntgendiagnostischen Untersuchungen auftreten, nicht geführt werden, sodass hierüber prinzipiell nicht aufgeklärt werden muss.

3. Die RöV beinhaltet eigenständige Maßstäbe für die Anwendung von Röntgenstrahlung am Menschen aus medizinischen Gründen, die sich auch auf die zivilrechtlichen Pflichten des Arztes im Rahmen des Behandlungsvertrages nach den $\S \S 630 \mathrm{a}$ ff. BGB auswirken.

4. Vor der Durchführung einer Röntgenuntersuchung ist die Stellung der rechtfertigenden Indikation durch ei- 
nen Arzt mit der erforderlichen Fachkunde im Strahlenschutz vorzunehmen.

5. Der Arzt hat die Feststellung zu treffen, dass das Ziel der Untersuchung den gesundheitlichen Nutzen gegenüber dem Strahlenrisiko bei dem konkreten Patienten überwiegt.

6. Bei der erforderlichen Abwägung hat der Arzt auch zu prüfen, ob andere Verfahren mit vergleichbarem gesundheitlichem Nutzen, die mit keiner oder einer geringeren Strahlenexposition verbunden sind, im konkreten Fall für den Patienten zur Verfügung stehen.

7. Die Stellung der rechtfertigenden Indikation ist nur zulässig, wenn der Arzt die Möglichkeit hat, den Patienten vor Ort persönlich zu untersuchen.

8. Eine persönliche Untersuchung durch den Arzt vor Anfertigen einer Röntgenaufnahme ist bei eindeutiger Rechtfertigung nicht erforderlich.
9. Zur Abklärung bestehender Risiken des Patienten können diese durch nicht ärztliches Personal nach $§ 24$ Abs. 2 RöV befragt werden.

10.Ergibt die Befragung des Patienten ein erhöhtes Risiko (z.B. Zwischenfälle bei einer früheren Untersuchung, Schwangerschaft) ist der Arzt zur persönlichen Aufklärung und Untersuchung des Patienten verpflichtet.

11.Die Risikoaufklärung nach $\S 630$ e BGB kann nicht an nicht ärztliche Fachkräfte delegiert werden und muss entweder von dem Radiologen oder einem anderen Arzt nach $\S 24$ Abs. 1 Nr. 3 RöV durchgeführt werden, der über die erforderlichen Kenntnisse im Strahlenschutz verfügt.
Prof. Dr. Peter Wigge Rechtsanwalt, Fachanwalt für Medizinrecht

Rechtsanwälte Wigge

Scharnhorststr. 40

48151 Münster

Telefon: (0251) 53 595-0

Telefax: (0251) 53 595-99

E-Mail: kanzlei@ra-wigge.de

Prof. Dr. Dr. Reinhard Loose Chefarzt, Institut für Radiologie Nord

Klinikum Nürnberg Nord Prof.-Ernst-Nathan-Str. 1 90419 Nürnberg

Tel: 0911-398-2540

Fax: 0911-398-2073

E-Mail: Reinhard.Loose@klinikum-nuernberg.de 